\title{
Predictability of the cue-target relation and the time-course of auditory inhibition of return
}

\author{
TODD A. MONDOR \\ Mount Allison University, Sackville, New Brunswick, Canada
}

\begin{abstract}
The possibility that the time-course of auditory inhibition of return (IOR) might depend on the temporal or spatial predictability of the cue-target relation was investigated. In all the experiments, a location cue was followed by a target that was to be localized. An inhibitory effect became apparent at a longer stimulus onset asynchrony (SOA) when either the temporal or the spatial relation was predictable, rather than when either was unpredictable. A facilitative effect was apparent at a 100-msec SOA, irrespective of the predictability of the cue-target relation. These results establish that the timecourse of the inhibitory component of location-based auditory IOR depends on the predictability of the temporal and spatial relations of cue and target. The theoretical implications of these results are considered, and a dual-process model of auditory selective attention is offered.
\end{abstract}

Audition was, at one time, the modality of choice with regard to presenting information to study selective attention, and early models were based largely on the results of such experiments (e.g., Broadbent, 1957; Deutsch \& Deutsch, 1963; Moray, 1974; Norman, 1968; Treisman, 1970). However, audition was soon replaced by vision as the preferred modality of study, with the result that, whereas much is now known about the selection of visual information, relatively little is known about the selection of auditory information. Interest in auditory selective attention has, however, been renewed in the last few years (e.g., Hafter, Schlauch, \& Tang, 1993; Mondor \& Bregman, 1994; Mondor \& Bryden, 1992; Mondor \& Zatorre, 1995; Rhodes, 1987; Scharf, Quigley, Aoki, Peachey, \& Reeves, 1987; Spence \& Driver, 1994; Woods, 1990). Much of this recent research has relied on a simple paradigm in which a single cue is presented, followed by a single target, about which listeners are required to make some type of detection or identification judgment. Usually, the time period between the onset of the cue and the onset of the target (stimulus onset asynchrony [SOA]) and the similarity of the cue and the target are examined for their effect on the speed and accuracy of responding to the target. This research has established, among other things, that the effect on performance of uninformative spatial and frequency cues varies as a function of SOA. Specifically, relative to performance for targets that differ from the preceding cue, performance for targets that are similar

This research was supported by a grant from the Natural Sciences and Engineering Research Council of Canada. I thank Bruce Schneider and Steve Joordens for their insightful criticism of an earlier version of this paper. Correspondence concerning this article should be addressed to T. A. Mondor, Department of Psychology, 49A York St., Mount Allison University, Sackville, NB, E4L 1C7 Canada (e-mail: tmondor@) mta.ca). to the cue is facilitated at brief SOAs and inhibited at longer SOAs (Mondor \& Breau, 1999; Mondor, Breau, $\&$ Milliken, 1998). This pattern does not appear to depend on general task requirements, since Mondor et al. (1998) have reported its existence for both detection and identification tasks. The transition from facilitation at brief SOAs to inhibition at more lengthy SOAs that is engendered by uninformative cues has been labeled inhibition of return (IOR) by those who have investigated the effect within the visual modality (e.g., Posner \& Cohen, 1984). A study reported by Mondor and Breau suggests that auditory IOR is not produced by response biases generated in response to information provided by the cue. In this study, either the spatial or the frequency relation between a cue and a target was manipulated in separate conditions. In contrast with other, similar investigations, listeners were required to identify the rise-time of the target (defined as the rate with which maximal amplitude was obtained). Evidence of both location-based and frequency-based auditory IOR was obtained, using both response times (RTs) and errors as dependent measures. Because the judgment required of listeners was independent of cue-target similarity in location or frequency, Mondor and Breau argued that the facilitative and inhibitory effects of spatial and frequency cues result primarily because of changes in perceptual sensitivity.

Whereas the existence of auditory IOR has now been demonstrated, little is known about the factors that might limit this effect. Unsurprisingly, given the more extensive investigation of visual attention, much more is known about the factors that limit visual IOR (for a review, see Klein \& Taylor, 1994). Indeed, it appears that a variety of factors modulate the strength of the phenomenon, including overt orienting (Maylor, 1985), saccade preparation and execution (Rafal, Calabresi, Brennan, \& Sciolto, 1989; Rafal, Egly, \& Rhodes, 1994), the number of si - 
multaneous location cues (Maylor, 1985; Posner \& Cohen, 1984), age (Clohessy, Posner, Rothbart, \& Vecera, 1991), and even personality (Avila, 1995).

In addition to these factors, the accuracy of the information provided by a cue has also been reported to influence visual IOR, with the inhibitory component apparently being eliminated when the likelihood that the cue provides accurate spatial information is better than chance (see, e.g., Klein \& Taylor, 1994; Rafal et al., 1989; Rafal \& Henik, 1994). The failure to identify an inhibitory cuing effect when there is a predictable spatial relation between cue and target has been attributed to the fact that an endogenous (voluntary) attentional mechanism controls selection in such situations (Müller \& Rabbitt, 1989). On the other hand, visual IOR, with its facilitative and inhibitory components, has usually been attributed to the operation of an exogenous (involuntary) attentional mechanism (Maylor \& Hockey, 1985).

With regard to auditory IOR, an inhibitory cuing effect has not been reported when spatial (Mondor \& Zatorre, 1995) or frequency (Mondor \& Bregman, 1994) predictability is high. In addition, Mondor and Amirault (1998) found that facilitative cross-modal spatial cuing effects depended, in part, on the overall predictability of the cue-target relation. This appeared to apply to both the modality and the temporal relations between cue and target, so that facilitative effects of different-modality spatial cues were more likely to occur when the modalities of the cue and the target were held constant and when only a single SOA was used. This investigation was limited to brief SOAs, and therefore, the effect of predictability on within-modality inhibitory effects was not addressed. Although certainly far from overwhelming, this evidence does suggest that predictability may be an important determinant of auditory IOR.

The present study was undertaken to examine the possible influence of predictability of the cue-target relation on auditory IOR, as a means of extending our understanding of the phenomenon. One prediction that may be derived from current thinking about visual IOR is that increasing predictability should act to increase the involvement of endogenous attentional processes and, thereby, either reduce the magnitude of or eliminate entirely any inhibitory cuing effect. To anticipate somewhat, this prediction was not supported, since the obtained results indicated that an inhibitory effect of about the same magnitude emerges at a longer SOA when there is a predictable relation between cue and target, rather than when there is not. The theoretical implications of these results are considered, and a dual-process model of auditory selective attention is offered to account both for these results and for other recent findings.

\section{GENERAL METHOD}

In all the experiments, an auditory spatial cue was followed by an auditory target. Listeners were required to make a speeded judgment regarding target location (left, right). Across experiments, either the number of SOAs possible within a block of trials or the probability that the target would sound in the cued location was manipulated, in order to determine whether location-based auditory IOR depends on the predictability of the temporal or the spatial relation between a spatial cue and a target.

\section{Subjects}

Undergraduate students attending Mount Allison Unıversity received course credit in exchange for their participation. All the listeners reported that they had normal, uncorrected hearing. None of the volunteers participated in more than one experiment.

\section{Materials}

Computer and sound system. A 486/50 desktop PC runnıng the MAPLE programming environment (Bregman, Achim, \& Ahad, 1992) was used to present sounds and to record responses. The sounds were presented over Sony SRS-PC30 speakers positıoned $45^{\circ}$ to the left and the right of the listener (speakers were approx1mately $50 \mathrm{~cm}$ from the listener's midline). The sounds were presented at approximately $65 \mathrm{~dB}$ SPL.

Sounds. A single pure tone of $1000 \mathrm{~Hz}, 50 \mathrm{msec}$ in duration, was synthesized at $32000 \mathrm{~Hz}$, using the MITSYN time-signal processing software package (Henke, 1990). The tone included 5-msec onset/offset amplitude ramps to eliminate clicks. This was the only sound used in the experiments.

\section{Design and Procedure}

On each trial of all the experiments, a location cue presented in either the left or the right location was followed by a target presented in either the left or the right location. The probability that the target would sound in the same location as (a repeat trial) or in a different location from (a change trial) the cue was .50 in Experiments 1 and 2 (i.e., there were an equal number of repeat and change trials) and .60 in Experiments 3 and 4 (i.e., there were exactly $60 \%$ repeat and $40 \%$ change trials). All the subjects were informed at the beginning of each experiment as to the probability that the cue and the target would be similar in location, as well as to the number of different SOAs possible. Listeners were required to localize each target and made their responses by pressing the 1 key on a computer keyboard for targets sounding from the left and the 0 key for targets sounding from the right (this mapping was reversed for half of the subjects in each experiment). Each experiment was selfpaced, so that the subject initiated each trial by pressing any key on the keyboard. Listeners were instructed (1) to look at the monitor directly in front of them and to refrain from moving their eyes and (2) to respond as quickly as possible, while at the same time avoiding errors. Both RTs and error rates were recorded. In all the experiments, mean RT was calculated for correct responses only after those RTs more than 2.5 standard deviations beyond the mean were eliminated. For each experiment, a table is presented delineating mean RT and error rate as a function of SOA.

\section{EXPERIMENT 1}

Experiment 1 consisted of an attempt to replicate and extend previous reports of location-based auditory IOR when a localization task is used and cue and target may be separated temporally by three different time periods (Mondor, Breau, \& Milliken, 1998). Three SOAs were used, but these covered a much larger range than was the case in previous studies. Specifically, the cue could precede the target by 100,700 , or $1,300 \mathrm{msec}$, rather than by 100,400 , and $700 \mathrm{msec}$, as was used by Mondor and Breau (1999) and Mondor, Breau, and Milliken (1998). Thus, neither the temporal nor the spatial relation between the cue and the target could be predicted with any 
Table 1

Response Times (RT, in Milliseconds, With Standard Errors) and Percent Errors (PE) for Repeat and Change Trials as a Function of Repetition and Stimulus Onset Asynchrony in Experiment 1

\begin{tabular}{|c|c|c|c|c|c|c|c|c|c|}
\hline \multirow[b]{3}{*}{ Trials } & \multicolumn{9}{|c|}{ Stimulus Onset Asynchrony } \\
\hline & \multicolumn{3}{|c|}{$100 \mathrm{msec}$} & \multicolumn{3}{|c|}{$700 \mathrm{msec}$} & \multicolumn{3}{|c|}{$1,300 \mathrm{msec}$} \\
\hline & $\mathrm{RT}$ & $S E$ & $\mathrm{PE}$ & RT & $S E$ & $\mathrm{PE}$ & RT & $S E$ & $\mathrm{PE}$ \\
\hline Repeat & 589 & 42.24 & 5.1 & 550 & 26.09 & 2.1 & 501 & 24.83 & 3.0 \\
\hline Change & 742 & 47.42 & 20.3 & 524 & 29.64 & 2.1 & 479 & 26.91 & 2.5 \\
\hline
\end{tabular}

degree of certainty. This experiment permitted determination of whether the SOA at which an inhibitory effect becomes apparent is dependent on the range of SOAs employed and whether an inhibitory effect at a $700-\mathrm{msec}$ SOA (if obtained) might persist at a 1,300-msec SOA. Eighteen listeners completed 36 practice and 144 experimental trials ( 24 for each combination of repetition and SOA).

\section{Results and Discussion}

Mean RT and error rate as a function of repetition and SOA are described in Table 1. Separate two-way withinsubjects analyses of variance (ANOVAs; repetition [repeat, change] $\times$ SOA $[100,700,1,300])$ were performed for the RT and error data. The RT analysis revealed significant main effects of repetition $[F(1,17)=10.45, p<$ $.01]$ and $\operatorname{SOA}[F(2,34)=44.20, p<.001]$. In addition, a significant interaction between repetition and SOA $[F(2,34)=27.15, p<.001]$ arose because a facilitative effect (i.e., advantage for repeat trials) at the 100-msec SOA reversed to an inhibitory effect (i.e., an advantage for change trials) at both the $700-$ and the $1,300-\mathrm{msec}$ SOA ( $p<.015$, for all three comparisons). The magnitude of these inhibitory effects did not differ from one another ( $p>.35$, for the comparisons).

In the analysis of the error data, ${ }^{2}$ the main effects of repetition $[F(1,17)=20.75, p<.001]$ and SOA $[F(2,34)=$ $31.09, p<.001]$ and the interaction $[F(2,34)=28.39$, $p<.001]$ were all significant. Comparisons of performance on repeat and change trials at each SOA indicated a significant facilitative effect at the $100-\mathrm{msec}$ SOA $(p<.001)$.

Although the range of SOAs used was larger than in previous studies, these results replicate previous reports of location-based auditory IOR (Mondor \& Breau, 1999; Mondor, Breau, \& Milliken, 1998; Reuter-Lorenz, Jha, \& Rosenquist, 1996; W. C. Schmidt, 1996). In addition, it appears that when the cue and the target may be separated by three different intervals, an inhibitory effect appears by $700 \mathrm{msec}$ and persists at least until the 1,300msec SOA. This pattern, obtained under conditions in which the listener was unable to predict either the temporal or the spatial relation between the cue and the target, will be used as the basis of comparison for the results reported in the next three experiments.

\section{EXPERIMENT 2}

In Experiment 2, three different groups of 18 subjects localized targets when only a single SOA was used. For each group, the cue preceded the target by either 100 , 700 , or $1,300 \mathrm{msec}$. In contrast with Experiment 1 , listeners were able to establish a precise temporal expectancy for the occurrence of the target, relative to the presentation of the cue. This design allowed, then, determination of whether the facilitative and inhibitory effects apparent in Experiment 1 are influenced by the temporal predictability of the cue-target relation. Listeners completed 12 practice and 144 experimental trials ( 72 repeat and 72 change trials).

\section{Results and Discussion}

Mean RTs and error rates for each SOA as a function of repetition are described in Table 2 .

100-msec SOA condition. Performance on repeat trials was significantly better than that on change trials for both RTs $[F(1,17)=18.61, p<.001]$ and errors $[F(1,17)=11.99, p<.001]$. Thus, a facilitative effect was apparent, even though uncertainty regarding the temporal relation between the cue and the target was minimized.

700-msec SOA condition. Whereas there was no difference in RTs on repeat and change trials $(F<1)$, a facilitative effect was apparent for the error data $[F(1,17)=$

Table 2

Response Times (RT, in Milliseconds, With Standard Errors) and Percent Errors (PE) at 100-, 700-, and 1,300-msec Stimulus Onset Asynchronies as a Function of Repetition in Experiment 2

\begin{tabular}{|c|c|c|c|c|c|c|c|c|c|}
\hline \multirow[b]{3}{*}{ Trials } & \multicolumn{9}{|c|}{ Stimulus Onset Asynchrony } \\
\hline & \multicolumn{3}{|c|}{$100 \mathrm{msec}$} & \multicolumn{3}{|c|}{$700 \mathrm{msec}$} & \multicolumn{3}{|c|}{$1,300 \mathrm{msec}$} \\
\hline & RT & $S E$ & $\mathrm{PE}$ & RT & $S E$ & $\mathrm{PE}$ & RT & $S E$ & $\overline{\mathrm{PE}}$ \\
\hline Repeat & 487 & 29.77 & 4.2 & 521 & 29.60 & 0.1 & 418 & 32.46 & 1.4 \\
\hline Change & 589 & 42.45 & 14.3 & 519 & 29.35 & 4.4 & 396 & 34.44 & 1.4 \\
\hline
\end{tabular}


5.47, $p<.05]$. Performance at a $700-\mathrm{msec}$ SOA, given no temporal ambiguity, then, obviously differed markedly from the inhibitory effect apparent at an equivalent SOA, given some uncertainty as to the temporal relation of the cue and the target. Indeed, a comparison of the effect of repetition at the 700-msec SOA in Experiments 1 and 2 indicated a statistically significant difference $(p<.01)$.

1,300-msec SOA condition. A significant inhibitory effect was apparent for RTs $[F(1,17)=15.89, p<.001]$. Although no such effect was obtained for errors $(F<1)$, this seems most likely to be the result of the very low error rate. Thus, an inhibitory effect of spatial cues on target localization performance was obtained, even though there was no variability in the temporal relation of the cue and the target. The magnitude of this inhibitory effect is not statistically different than those apparent at the 700- and 1,300-msec SOAs in Experiment $1(p>.25$, for both comparisons).

Taken together, the results of Experiments 1 and 2 suggest that the inhibitory effect of a location cue on target discrimination is dependent on the temporal predictability of the cue-target relation. Specifically, given a highly predictable temporal relation between a cue and a target, the inhibitory component of auditory IOR does occur, but at a more lengthy SOA than is the case with a less predictable relation.

\section{EXPERIMENT 3}

Experiments 1 and 2 appear to provide quite strong evidence that the temporal predictability of the cue-target relation affects the time-course of auditory IOR. Critically, increasing temporal predictability acts to delay the appearance of inhibition but does not seem to reduce its magnitude. This modulation of the time-course of auditory IOR raises the possibility that the predictability of other characteristics of the cue-target relation might also be important in determining the effect of location cues on target discrimination performance. This possibility was addressed in Experiment 3, wherein the spatial predictability of the cue and the target relation was changed from that used in the previous experiments. It has been reported that neither visual nor auditory IOR is apparent when there is a predictable spatial relation between a cue and a target (e.g., Rafal \& Henik, 1994). However, the empirical evidence on which these claims have been made appears to have been based on studies or observations of performances with a quite robust predictable re- lation of .75 or more. For example, Mondor and Zatorre (1995, Experiment 2) reported no evidence of an inhibitory cuing effect at SOAs of up to $1,500 \mathrm{msec}$ when the probability that an auditory target would be presented in the same location as a preceding spatial cue was approximately .86 .

The possibility that a moderate increase in spatial predictability might alter the time-course of auditory IOR was investigated by increasing the probability that the target would sound from the same location as the cue from .50 to .60 . Thus, the probability of a repeat trial was .60 , and the probability of a change trial was .40 . The cue could precede the target by $100,700,1,300$, or $1,900 \mathrm{msec}$. The listeners completed 20 practice and 240 experimental trials ( 36 repeat trials and 24 change trials per SOA).

\section{Results and Discussion}

Mean RTs and error rates as a function of repetition and SOA are described in Table 3. A two-way withinsubjects ANOVA (repetition [repeat, change] $\times$ SOA $[100,700,1,300,1,900 \mathrm{msec}])$, using RT as the dependent measure, indicated that whereas the main effect of SOA $[F(3,33)=56.52, p<.001]$ reached significance, the main effect of repetition $[F(1,11)=1.57, p=.23]$ did not. The significant interaction between repetition and SOA $[F(3,33)=14.67, p<.001]$ resulted because a significant facilitative effect at the $100-\mathrm{msec}$ SOA reversed to a significant inhibitory effect at the 1,900 msec SOA ( $p<.05$, for both comparisons). The magnitude of this inhibitory effect was not significantly different from any of those reported in Experiments 1 and 2 . The difference between repeat and change trials in mean RT did not approach significance at either of the other SOAs ( $p>.21$, for both comparisons). Although not statistically significant, the magnitude of the 26-msec inhibitory effect at the 1,300 -msec SOA was quite similar to the 22-msec effect at the same SOA that was apparent in Experiment 2. This similarity arose because of large inhibitory effects (118 and $172 \mathrm{msec}$ ) on the part of 2 listeners in the present experiment. That only 7 out of 12 listeners showed an inhibitory effect at the $1,300-\mathrm{msec}$ SOA in this experiment (as compared with 11 out of 12 in Experiment 2) would appear to indicate that there was not any systematic effect of repetition at $1,300 \mathrm{msec}$ in the present experiment.

For the error data, the main effects of repetition $[F(1,11)=45.52, p<.001]$ and $\operatorname{SOA}[F(3,33)=17.43$, $p<.001]$ and the interaction between the two variables

Table 3

Response Times (RT, in Milliseconds, With Standard Errors) and Percent Errors (PE) as a Function of Repetition and Stimulus Onset Asynchrony in Experiment 3 Stimulus Onset Asynchrony

\begin{tabular}{|c|c|c|c|c|c|c|c|c|c|c|c|c|}
\hline \multirow[b]{3}{*}{ Trials } & \multicolumn{12}{|c|}{ Stimulus Onset Asynchrony } \\
\hline & \multicolumn{3}{|c|}{$100 \mathrm{msec}$} & \multicolumn{3}{|c|}{$700 \mathrm{msec}$} & \multicolumn{3}{|c|}{$1,300 \mathrm{msec}$} & \multicolumn{3}{|c|}{$1,900 \mathrm{msec}$} \\
\hline & $\mathrm{RT}$ & $S E$ & $\mathrm{PE}$ & $\mathrm{RT}$ & $S E$ & PE & $\mathrm{RT}$ & $S E$ & $\mathrm{PE}$ & RT & $S E$ & PE \\
\hline Repeat & 662 & 39.07 & 3.4 & 581 & 29.84 & 2.0 & 582 & 33.67 & 1.1 & 542 & 26.42 & 1.5 \\
\hline Change & 808 & 48.29 & 14.4 & 584 & 36.92 & 1.9 & 556 & 34.45 & 2.5 & 513 & 28.90 & 1.6 \\
\hline
\end{tabular}

Note-In this experiment, the probability of a repeat trial was .6 , and the probability of a change trial was .4 . 
$[F(3,33)=11.40, p<.001]$ all reached significance. Planned comparisons revealed a facilitative effect at the 100 -msec SOA $(p<.01)$, with no difference between repeat and change trials at any other SOA $(p>.14$, in all cases).

Establishing a moderate predictive relation between the cue and the target delayed the appearance of inhibition until the $1,900-\mathrm{msec}$ SOA. This is obviously substantially later than the 700-msec SOA at which inhibition was obtained in Experiment 1, wherein there was no such predictive spatial relation and multiple SOAs were used. Interestingly, reducing the unpredictability of the spatial relation even by only $10 \%$ delayed inhibition at least as much as did eliminating all uncertainty as to the temporal relation between the cue and the target. This difference may be related to the evidence, reported by Mondor, Breau, and Milliken (1998), that auditory selective attention is heavily dependent on location and frequency. In the terminology of Tsal and Lavie (e.g., 1993), who argued that location is a dominant feature controlling the selection of visual information, location and frequency appear to be co-dominant in guiding the selection of auditory information.

\section{EXPERIMENT 4}

The results of Experiment 3 establish that spatial predictability, like temporal predictability, affects the timecourse of auditory IOR. For both attributes, as predictability increased, the SOA at which inhibition emerged increased as well. One obvious issue that arises from these results is whether the effect of temporal and spatial predictability may be additive in determining the emergence of inhibition. If so, reducing temporal ambiguity while at the same time maintaining a predictable spatial relation between the cue and the target should result in an even greater delay of the inhibitory effect than has yet been observed. Alternatively, if the effects of temporal and spatial predictability are independent, the SOA at which inhibition emerges should be determined by the characteristic that alone imposes the greatest delay. Given such independence, the results of Experiments 1-3 would suggest that when an unambiguous temporal relation is coupled with a moderately predictable spatial relation, spatial predictability would dictate performance and the inhibitory effect would emerge at about the 1,900-msec SOA, just as it did in Experiment 3 (assuming, of course, that the nonsignificant inhibitory effect at the 1,300-msec SOA in Experiment 3 was not indicative of a systematic effect). Experiment 4 was designed to distinguish between these possibilities. To this end, spatial predictability was maintained at .60 , but only a single SOA was used. For different groups of 12 subjects, the cue could precede the target by either a 1,900or a 2,500-msec SOA. Performance at brief SOAs was not examined in this study, because previous research has established that a facilitative effect is apparent both
Table 4

Response Times (RT, in Milliseconds, With Standard Errors) and Percent Errors (PE) at 1,900- and 2,500-msec Stimulus Onset Asynchronies as a Function of Repetition in Experiment 4

\begin{tabular}{|c|c|c|c|c|c|c|}
\hline \multirow[b]{3}{*}{ Trials } & \multicolumn{6}{|c|}{ Stimulus Onset Asynchrony } \\
\hline & \multicolumn{3}{|c|}{$1,900 \mathrm{msec}$} & \multicolumn{3}{|c|}{$2,500 \mathrm{msec}$} \\
\hline & RT & $S E$ & $\mathrm{PE}$ & RT & $S E$ & $\mathrm{PE}$ \\
\hline Repeat & 430 & 22.97 & 0.7 & 404 & 21.15 & 1.9 \\
\hline Change & 438 & 24.23 & 1.2 & 392 & 21.90 & 1.4 \\
\hline
\end{tabular}

Note- - In this experiment, the probability of a repeat trial was .6, and the probability of a change trial was .4 .

at $60-\mathrm{msec}$ and at 600 -msec SOAs when there is a predictable spatial ( $75 \%$ repeat trials) and temporal (one SOA) relation between a cue and a target (Mondor \& $\mathrm{Za}-$ torre, 1995, Experiments 4 and 5). The listeners completed 20 practice and 160 experimental trials ( 96 repeat trials and 64 change trials).

\section{Results and Discussion}

Mean RTs and error rates for each SOA as a function of repetition are described in Table 4.

1,900-msec SOA. Performance on repeat and change trials did not differ in either RT $[F(1,11)=2.07, p=.18]$ or error rate $(F<1)$. This null effect of repetition differed significantly from the inhibitory effect apparent at the 1,900-msec SOA in Experiment $3(p<.01)$.

2,500-msec SOA. As is shown in Table 4, whereas the two trial types did not differ in accuracy $(F<1)$, an inhibitory effect in RT was apparent $[F(1,11)=5.57, p<$ $.05]$. It appears, then, that increasing both spatial and temporal predictability acts to delay the appearance of an inhibitory cuing effect more than does increasing the predictability of either one of these characteristics alone. Thus, as is shown in Figure 1, the effect of predictability seems to be quantitative, with the magnitude of the effect being a function of the degree of predictability on multiple characteristics (at least for the two characteristics investigated in this study).

\section{GENERAL DISCUSSION}

Theoretical accounts of visual IOR have focused on the potential adaptive value that inhibiting attention from returning to a previously attended location might have with regard to obtaining new information from the environment (e.g., Houghton \& Tipper, 1994; Posner \& Cohen, 1984). This explanation is typically associated with the theoretical assumption that the act of attending and the act of ignoring are intimately related, usually as different outcomes of the same underlying mechanism(s). As Houghton and Tipper have noted, this assumption is quite consistent with several pieces of empirical evidence, particularly as reported by Maylor (1985), who found that overt orienting eliminated both facilitative and inhibitory cuing effects and that simulta- 


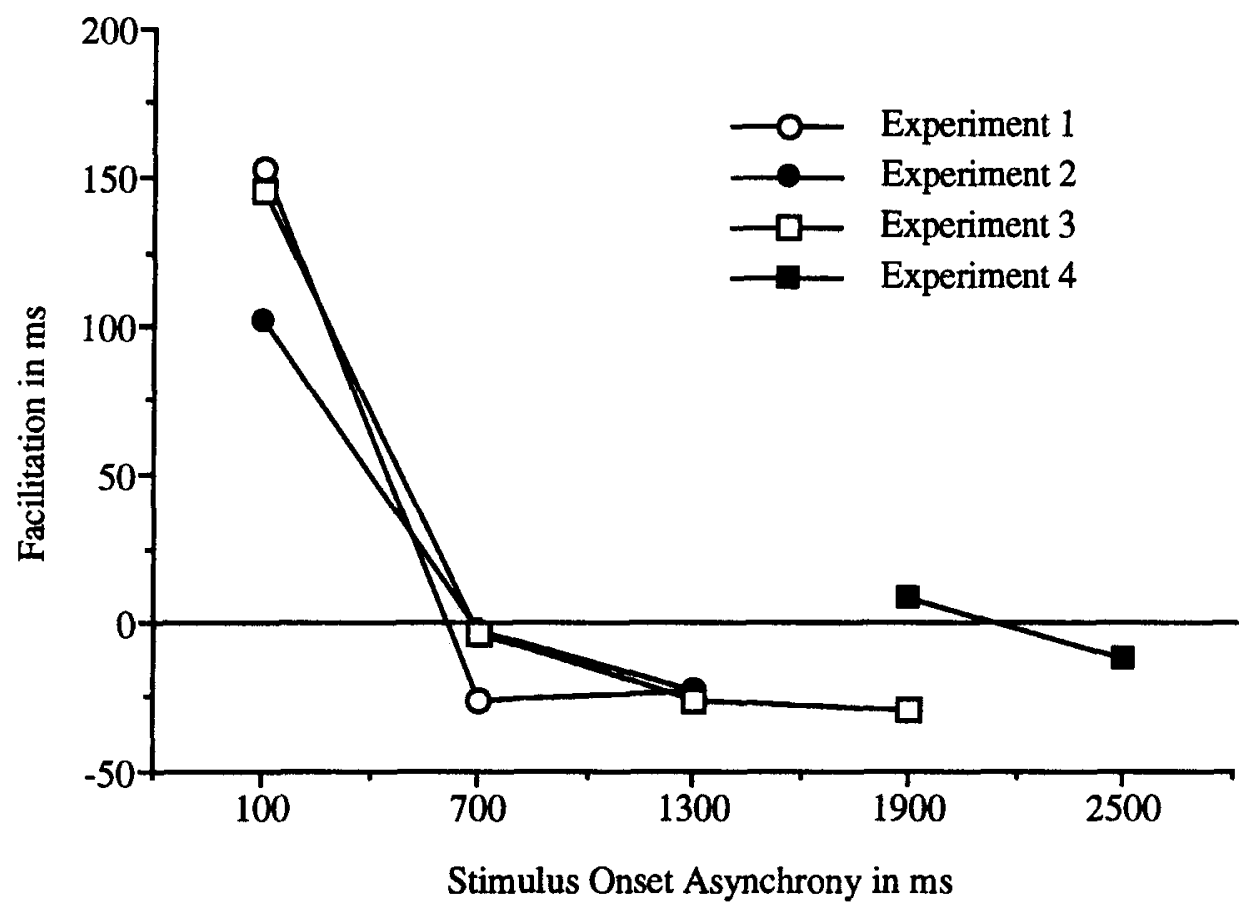

Figure 1. The facilitative effect at each stimulus onset asynchrony in all experiments. Note that negative numbers indicate an inhibitory effect.

neously cuing two locations reduced by approximately $50 \%$ the magnitude of both facilitative and inhibitory effects (but see Posner \& Cohen, 1984, who found that such double-cuing eliminated facilitation but did not affect inhibition). In contrast, the results of the present series of experiments, showing that predictability substantially influences the time-course of only the inhibitory component of auditory IOR, appears to be inconsistent with this general viewpoint and suggests that there is at least a partial independence of the mechanisms responsible for facilitation and those responsible for inhibition. Thus, the effect of predictability on the time-course of auditory IOR may be quite informative with respect to establishing a theoretical explanation. The connectionist model of visual selective attention proposed by Houghton and Tipper will be taken as a starting point with respect to evaluating the implications of these results, both because it incorporates an explicit account of visual IOR and because it appears to be quite consistent with much of the empirical evidence regarding visual covert orienting.

Houghton and Tipper (1994) suggested that selection is accomplished by the interaction of four basic structures/ mechanisms, the operation of which were described within a connectionist framework. The object field represents the product of a preattentive process that acts to construct integrated representations of objects, using basic visual features. The target field acts as an attentional template representing current goals. The match/ mismatch field represents the operation of a process that, in effect, monitors the match between the target field and the object representations in the object field. In the connectionist terminology of the model, the connection weights of visual objects are modified depending on the extent to which they match the target field, with the net result being that representations that closely match the target field are excited and those that do not match the target field are inhibited. Finally, response binding allows the appropriate response or response schema to be linked to the target representation.

An explicit assumption on which the model proposed by Houghton and Tipper (1994) is founded is that facilitation and inhibition arise because of the operation of the same underlying process. That is, there is a "coupling of facilitatory and inhibitory components of IOR, such that the inhibitory component manages to 'hit' just what was previously facilitated" (Houghton \& Tipper, 1994, p. 100). In practical terms, this assumption would appear to require that any manipulation that affects inhibition should also affect facilitation, and in the same way. Were this true for auditory IOR, one might have expected that predictability would delay the emergence of both facilitation and inhibition. This was clearly not the case, since a robust facilitative effect was apparent at a $100-\mathrm{msec}$ SOA, irrespective of the temporal or spatial predictability of the cue-target relation. The obtained dissociation suggests the possibility that facilitation and inhibition represent the operation of at least partially different processes or mechanisms (see, also, Posner \& Cohen, 
1984). Thus, whereas the Houghton and Tipper model is consistent with much of what is known about visual selective attention, as it is currently instantiated, it does not appear that the model can explain the dependence of the time-course of auditory IOR on the temporal and spatial predictability of the cue-target relation. To be fair, the model was not and could not have been designed to account for this effect.

One of the fundamental characteristics of the match/ mismatch field component of the Houghton and Tipper (1994) model is that it consists of a variety of subfields, each coding for a specific property, such as color or location. Therefore, "no signals are generated from match/ mismatch subfields which code for properties of a different type than the target property" (p. 73). The implication here appears to be that differences between the cue and the target in properties that are irrelevant to the task at hand should neither enhance nor diminish the magnitude of facilitative and inhibitory cuing effects. The obtained effect of temporal predictability is, of course, inconsistent with this proposal.

\section{The Dual-Process Model}

In this paper, I would like to suggest a way of thinking about auditory selective attention that is consistent with both the existence of auditory IOR and with the effect of predictability obtained in this study. This model is derived from an account of facilitative auditory cuing effects proposed by Mondor, Zatorre, and Terrio (1998), which itself was developed using elements of both Houghton and Tipper's (1994) model and the attentional engagement theory proposed by Duncan and Humphreys (1989, 1992). Briefly, Mondor, Zatorre, and Terrio (1998) suggested that selection operates on auditory objects, or streams, which are constructed by a scene analysis process such as that proposed by Bregman (e.g., 1990). Selection is accomplished via an attentional template, which may be thought of as an advance description. The parameters of this template may be set either bottom-up by the features of an advance cue or top-down in accordance with a predefined goal or strategy. On the basis of their observations that location and frequency may not be attended separately and that the location and the frequency information provided by an advance cue may not be used independently to guide selection, Mondor, Zatorre, and Terrio (1998) suggested that the attentional template is normally defined only by location and frequency. Recent evidence, reported by Zatorre, Mondor, and Evans (in press), that the same brain regions are active whether sounds are selected on the basis of location or of frequency appears to provide additional support for this proposal.

According to this account, the degree to which the target representation matches the attentional template determines the speed with which this representation reaches an auditory short-term memory store, where the appropriate response is determined. The better the match, the faster the selection and, all other things being equal, the more quickly the response assignment process may begin Thus, a facilitative cuing effect results because selection is faster when the location and/or the frequency of a target matches that of a preceding cue (as on a repeat trial) than when there is a mismatch (as on a change trial). Because facilitative effects of noninformative auditory spatial cues seem to disappear within about $300 \mathrm{msec}$, Mondor et al. suggested that, given a noninformative cue, specification of the template dissipates over roughly the same time period.

Presumably, when a listener is presented with a cue followed by a target, representations of both events are maintained in memory for some period of time. At the time of response assignment, then, it is likely that a distinction must be made between cue and target memory representations. On a repeat trial, the cue and the target representations are identical, except for the time of occurrence, and therefore, one might expect that this distinction will be relatively difficult. In contrast, on a change trial, the cue and the target representations will differ not only in the time of occurrence, but also on a second feature (usually the variable of interest-location, in the present experiments), and one might expect that differentiating the two memory representations will be relatively easy. Thus, it seems reasonable to infer that, on average, more time will be required to differentiate the representation of the target from that of the cue on a repeat trial than on a change trial (see, e.g., Hunt, 1995; S. R. Schmidt, 1991). It is likely that the magnitude of this disadvantage for repeat trials will decline relatively slowly over time as the difference between the cue and the target in time of occurrence increases and this becomes a more salient cue for differentiation.

\section{An Account of Auditory Inhibition of Return}

The dual-process model, then, suggests that performance in the typical cue-target experiment may be affected by two processes - namely, attentional template specification and memory representation differentiation. With regard to template specification, the better the match between the template (as defined bottom-up by the location and frequency of the cue) and the target, the faster selection will be accomplished. With regard to distinguishing memory representations, the more similar the representations of the cue and the target, the more slowly differentiation will be accomplished. If attentional template specification exerts a relatively greater influence over performance at brief SOAs and memory representation differentiation exerts relatively more influence over performance at longer SOAs, a novel account of auditory IOR naturally emerges. Specifically, at brief SOAs, the better match between the target representation and the attentional template on repeat trials yields a selection speed advantage. Because, at brief SOAs, template specification has a greater influence on performance than does representation differentiation, this advantage is expressed as a facilitative effect. As SOA increases, the influence on performance of the target- 
template match declines because the template definition dissipates. Therefore, the relative difficulty of differentiating cue and target memory representations becomes relatively more important in determining performance. At more lengthy SOAs, then, the greater difference between cue and target memory representations on change trials yields a differentiation speed advantage that is expressed as an inhibitory effect.

\section{An Explanation of the Effects of Predictability}

Consider now a situation in which a listener is informed that a target will almost certainly be presented in the same location as an advance cue. Given such a situation, it would be reasonable to expect a performance advantage for repeat trials at all possible SOAs (indeed, as was mentioned above, Mondor \& Zatorre, 1995, observed facilitation for SOAs of up to $1,500 \mathrm{msec}$ when there was a predictable spatial relation between a cue and a target). One way of representing this in the model would be if knowledge of the cue-target relation were used to extend the duration of the template. This could account for the present results, because an increase in template duration would effectively introduce a delay in the point at which the relative influence of memory representation differentiation exceeds that of the template and, thereby, produce a concomitant delay in the expression of inhibition. Furthermore, one might expect that this increase in the duration of the template description would be more pronounced when either location or frequency was predictable, since these two features appear to be co-dominant in the control of selection (Mondor, Zatorre, \& Terrio, 1998). The effect of predictability, then, may be represented in the model as indicative of the contribution of an endogenous attentional process that acts in a top-down fashion to lengthen the effective duration of the template.

\section{Summary}

The experiments reported above provide strong evidence that the temporal and spatial predictability of the cue-target relation significantly modulates the timecourse of auditory IOR. Although in its early stages of development, the dual-process model of auditory selective attention can account for this effect and can explain the existence of both location-based and frequencybased auditory IOR. Most important, the model generates testable predictions and should, therefore, be useful in guiding future research in productive directions. For these reasons, it would appear to provide a promising foundation on which to develop a more comprehensive theory.

\section{REFERENCES}

AVILA, C. (1995). Facilitation and inhibition of visual orienting as a function of personality. Personality \& Individual Differences, 18, 503-509. Bregman, A. S. (1990). Auditory scene analysis. London: MIT Press. Bregman, A. S., Achim, A., \& Ahad, P. A. (1992). The MAPLE software system [Computer software]. McGill University, Department of Psychology.
BroadBeNt, D. E. (1957). A mechanical model for human attention and memory. Psychological Review, 64, 205-215.

Clohessy, A. B., Posner, M. I., Rothbart, M. K., \& Vecera, S. P. (1991). The development of inhibition of return in early infancy. Journal of Cognitive Neuroscience, 3, 345-350.

Deutsch, J. A., \& Deutsch, D. (1963). Attention: Some theoretical considerations. Psychological Review, 70, 80-90.

DUNCAN, J., \& HUMPHREYS, G. (1989). Visual search and stimulus similarity. Psychological Review, 96, 433-458.

DUNCAN, J., \& HumphrEYS, G. (1992). Beyond the search surface: Visual search and attentional engagement. Journal of Experimental Psychology: Human Perception \& Performance, 18, 578-588.

Hafter, E. R., Schlauch, R. S., \& TANG, J. (1993). Attending to auditory filters that were not stimulated directly. Journal of the Acoustical Society of America, 94, 743-747.

HENKE, W. L. (1990). An interactive dialogue language for time signal processing [Computer software]. MIT Research Laboratory of Electronics, Cambridge, MA.

Houghton, G., \& TiPPER, S. P. (1994). A model of inhibitory mechanisms in selective attention. In D. Dagenbach \& T. H. Carr (Eds.), Inhibitory processes in attention, memory, and language (pp. 52-112). Toronto: Academic Press.

HUNT, R. R. (1995). The subtlety of distinctiveness: What von Restorff really did. Psychonomic Bulletin \& Review, 2, 105-112.

KLEIN, R. M., \& TAYLOR, T L. (1994). Categories of cognitive inhibition with reference to attention. In D. Dagenbach \& T. H. Carr (Eds.), Inhibitory processes in attention, memory, and language (pp. 113150). Toronto: Academic Press.

MAYLOR, A. M. (1985). Facilitory and inhibitory components of or1enting in visual space. In M.I. Posner \& O. S. M. Marın (Eds.), Attention \& performance $X I$ (pp. 189-204). Hillsdale, NJ: Erlbaum.

MAYLOR, A. M., \& HOCKEY, R. (1985). Inhibitory component of externally controlled covert orienting in space. Journal of Experimental Psychology. Human Perception \& Performance, 11, 777.787

MondOR, T. A., \& Amirault, K. J. (1998). Effect of same- and different-modality spatial cues on auditory and visual target identıfication. Journal of Experimental Psychology: Human Perception \& Performance, 24, 745-755.

MONDOR, T. A., \& BrEAU, L. M. (1999). Facilitative and inhibitory effects of location and frequency cues: Evidence of a modulation in perceptual sensitivity. Perception \& Psychophysics, 61, 438-444.

Mondor, T. A., BreaU, L. M., \& Milliken, B. (1998). Inhibitory processes in auditory selective attention: Evidence of location-based and frequency-based inhibition of return. Perception \& Psychophysics, 60, 296-302.

Mondor, T. A., \& Bregman, A. S. (1994). Allocating attention to frequency regions. Perception \& Psychophysics, 56, 268-276.

Mondor, T. A., \& BRYDEN, M. P. (1992). On the relation between auditory spatial attention and auditory perceptual asymmetries. Perception \& Psychophysics, 52, 393-402.

MONDOR, T. A., \& ZATORRE, R. J. (1995). Shifting and focusing auditory spatial attention. Journal of Experimental Psychology: Human Perception \& Performance, 21, 387-409.

Mondor, T. A., Zatorre, R. J., \& Terrio, N. A. (1998). Constraints on the selection of auditory information. Journal of Experimental Psychology: Human Perception \& Performance, 24, 66-79.

MoORE, B. C. J. (1989). An introduction to the psychology of hearing (3rd ed). Toronto: Academic Press

MoraY, N. (1974). A data base for theories of selective listening. In P. M. A. Rabbitt \& S. Dornic (Eds.), Attention and performance $V$ (pp. 119-135). New York: Academic Press.

Müller, H. J., \& RabBitT, P. M. A. (1989). Reflexive and voluntary orienting of visual attention: Time course of activation and resistance to interruption. Journal of Experimental Psychology. Human Perception \& Performance, 15, 315-330.

Norman, D. A. (1968). Toward a theory of memory and attention. Psychological Review, 75, 522-536.

PoSNER, M. I., \& COHEN, Y. (1984). Components of visual orientıng. In H. Bouma \& D. G. Bowhuis (Eds.), Attention and performance $X$ Control of language processes (pp. 531-555). Hillsdale, NJ: Erlbaum. Rafal, R. D., Calabresi, P. A., Brennan, C. W., \& Sciolto, T. K. 
(1989). Saccade preparation inhibits reorienting to recently attended locations. Journal of Experimental Psychology: Human Perception \& Performance, 15, 673-685.

RafAL, R. D., EGLY, R., \& RHODES, D. (1994). Effects of inhibition of return on voluntary and visually guided saccades. Canadian Journal of Experimental Psychology, 48, 284-300.

RaFAL, R. [D.], \& HeNIK, A. (1994). The neurology of inhibition: Integrating controlled and automatic processes. In D. Dagenbach \& T. H. Carr (Eds.), Inhibitory processes in attention, memory, and language (pp. 1-51). Toronto: Academic Press.

Reuter-Lorenz, P. A., JhA, A. P., \& Rosenquist, J. N. (1996). What is inhibited in inhibition of return? Journal of Experimental Psychology: Human Perception \& Performance, 22, 367-378.

RHODES, G. (1987). Auditory attention and the representation of spatial information. Perception \& Psychophysics, 42, 1-14.

Scharf, B., Quigley, S., Aoki, C., Peachey, N., \& Reeves, A. (1987). Focused auditory attention and frequency selectivity. Perception \& Psychophysics, 42, 215-223.

SCHмidt, S. R. (1991). Can we have a distinctive theory of memory? Memory \& Cognition, 19, 523-542.

SchmidT, W. C. (1996). Inhibition of return without visual input. Neuropsychologia, 34, 943-952.

SPENCE, C., \& DRIVER, J. (1994). Covert spatial orienting in audition: Exogenous and endogenous mechanisms. Journal of Experimental Psychology: Human Perception \& Performance, 20, 555-574.

Stevens, S. S., \& Newman, E. B. (1936). The localization of actual sources of sound. American Journal of Psychology, 1, 297-306.

Treisman, A. M. (1970). Contextual cues in dichotic listening. Quarterly Journal of Psychology, 12, 242-248.
TSAL, Y., \& LAVIE, N. (1993). Location dominance in attending to color and shape. Journal of Experimental Psychology. Human Perception \& Performance, 19, 131-139.

Woods, D. L. (1990). The physiological basis of selective attention: Implications of event-related potential studies. In J. W. Rohrbaugh, R. Parasuraman, \& R. Johnson, Jr. (Eds.), Event-related potentials (pp. 178-209). New York: Oxford University Press.

Zatorre, R. J., Mondor, T. A., \& Evans, A. C. (in press). Functional activation of right parietal and frontal regions during auditory attention to space and frequency. Neurolmage.

\section{NOTES}

1. A pure tone was used as cue and target in this experiment in order to facilitate future comparison of the results obtained in these experiments with those from experiments dealing with frequency-based inhibitory effects (e.g., Mondor, Breau, \& Milliken, 1998).

2. Although the error rate seems to be quite high at the $100-\mathrm{msec}$ SOA, this would appear to be consistent with previous evidence that pure tones of $1000 \mathrm{~Hz}$ are relatively more difficult to localize than complex tones or noise bursts (see, e.g., Moore, 1989; Stevens \& Newman, 1936). Pure tones of $1000 \mathrm{~Hz}$ were maintained as cue and target in all the experiments in order to facilitate comparison of results across experiments.

(Manuscript received February 17, 1998; revision accepted for publication October 8, 1998.) 